\title{
Impact of early diagnosis on functional disability in rheumatoid arthritis
}

\author{
Dam Kim ${ }^{1,2}$, Chan-Bum Choi ${ }^{1,2}$, Jiyoung Lee ${ }^{2}$, Soo-Kyung Cho ${ }^{1,2}$, Soyoung Won ${ }^{2}$, So-Young Bang ${ }^{3}$, \\ Hoon-Suk Cha ${ }^{4}$, Jung-Yoon Choe ${ }^{5}$, Won Tae Chung, ${ }^{6}$, Seung-Jae Hong7, Jae-Bum Jun ${ }^{1}$, Young Ok Jung ${ }^{8}$, \\ Jinseok Kim ${ }^{9}$, Seong-Kyu Kim ${ }^{5}$, Tae-Hwan Kim ${ }^{1}$, Tae-Jong Kim ${ }^{10}$, Eunmi Koh ${ }^{4}$, Hye-Soon Lee \\ Jaejoon Lee ${ }^{4}$, Jisoo Lee ${ }^{11}$, Sang-Heon Lee ${ }^{12}$, Shin-Seok Lee ${ }^{10}$, Sung Won Lee ${ }^{6}$, Seung-Cheol Shim ${ }^{13}$, \\ Dae-Hyun Yoo $^{1}$, Bo Young Yoon ${ }^{14}$, Yoon-Kyoung Sung ${ }^{1,2}$, Sang-Cheol Bae ${ }^{1,2}$, and for the KORONA \\ investigators $^{2}$
}

Department of Rheumatology, ${ }^{1}$ Hospital for Rheumatic Diseases, Hanyang University College of Medicine, Seoul; ${ }^{2}$ Clinical Research Center for Rheumatoid Arthritis (CRCRA), Seoul; ${ }^{3}$ Hanyang University Guri Hospital, Guri; ${ }^{4}$ Samsung Medical Center, Sungkyunkwan University School of Medicine, Seoul; ${ }^{5}$ Catholic University of Daegu School of Medicine, Daegu; ${ }^{6}$ Dong-A University Hospital, Busan; ${ }^{7}$ Kyung Hee University Medical Center, Seoul; ${ }^{8} \mathrm{Hallym}$ University Kangnam Sacred Heart Hospital, Seoul; ${ }^{9}$ Jeju National University Hospital, Jeju; ${ }^{10}$ Chonnam National University Hospital, Gwangju; ${ }^{11}$ Ewha Womans University Mokdong Hospital, Seoul; ${ }^{12}$ Konkuk University Medical Center, Seoul; ${ }^{13}$ Chungnam National University Hospital, Daejeon; ${ }^{14}$ Inje University Ilsan Paik Hospital, Goyang, Korea

Received: November 10, 2015 Revised : December 23, 2015 Accepted: April 15, 2016

\section{Correspondence to}

Sang-Cheol Bae, M.D.

Department of Rheumatology, Hospital for Rheumatic Diseases, Hanyang University College of Medicine, 222-1 Wangsimni-ro, Seongdong-gu, Seoul 04763, Korea Tel: +82-2-2290-9237

Fax: +82-2-2298-8231

E-mail: scbae@hanyang.ac.kr
Background/Aims: To determine whether early diagnosis is beneficial for functional status of various disease durations in rheumatoid arthritis (RA) patients. Methods: A total of 4,540 RA patients were enrolled as part of the Korean Observational Study Network for Arthritis (KORONA). We defined early diagnosis as a lag time between symptom onset and RA diagnosis of $\leq 12$ months, whereas patients with a longer lag time comprised the delayed diagnosis group. Demographic characteristics and outcomes were compared between early and delayed diagnosis groups. Logistic regression analyses were performed to identify the impact of early diagnosis on the development of functional disability in RA patients. Results: A total of 2,597 patients (57.2\%) were included in the early diagnosis group. The average Health Assessment Questionnaire-Disability Index (HAQ-DI) score was higher in the delayed diagnosis group ( $0.64 \pm 0.63$ vs. $0.70 \pm 0.66, p<0.01)$, and the proportion of patients with no functional disability $(\mathrm{HAQ}=0)$ was higher in the early diagnosis group (22.9\% vs. $20.0 \%, p=0.02)$. In multivariable analyses, early diagnosis was independently associated with no functional disability (odds ratio [OR], 1.19; 95\% confidence interval [CI], 1.01 to 1.40). In a subgroup analysis according to disease duration, early diagnosis was associated with no functional disability in patients with disease duration < 5 years (OR, 1.37; 95\% CI, 1.09 to 1.72) but not in patients with longer disease duration (for 5 to 10 years: OR, 1.07; 95\% CI, 0.75 to 1.52 ; for $\geq 10$ years: OR, $0.92 ; 95 \%$ CI, 0.65 to 1.28 ).

Conclusions: Early diagnosis is associated with no functional disability, especially in patients with shorter disease duration.

Keywords: Early diagnosis; Disability; Arthritis, rheumatoid; Health assessment questionnaires 


\section{INTRODUCTION}

During the past decade, early diagnosis and treatment of rheumatoid arthritis (RA) have been emphasized because a window of opportunity is believed to exist in early RA during which the disease is more responsive to treatment [1-3]. Previous studies revealed that early diagnosis and treatment prevent radiologic progression of RA and predict a greater rate of remission than does delayed treatment [4-6]. A meta-analysis of the literature showed that long-term progression of radiographic damage was significantly less frequent in patients treated with early disease-modifying anti-rheumatic drugs (DMARDs) than in patients receiving delayed treatment [6]. In addition, many studies have revealed that early intervention with DMARDs leads to a greater rate of remission than delayed intervention $[7,8]$.

Although functional outcome is as important as disease activity and radiologic progression, only a few studies have investigated the influence of early treatment on functional outcome [8-11]. Moreover, these studies focused on patients with relatively short disease duration, between 1 to 3 years, and the effect of early diagnosis and treatment on functional outcome according to disease duration is not clear.

In this study, the aim was to determine whether early diagnosis and treatment have benefits with respect to functional status with various disease durations in patients with RA.

\section{METHODS}

\section{Study population}

All RA patients aged 18 or older who satisfied the 1987 American College of Rheumatology criteria for RA [12] and who planned to have their blood drawn for routine evaluation were asked to provide informed consent and more than $95 \%$ of patients who were requested consented to participate in Korean Observational Study Network For Arthritis (KORONA) cohort [13]. A total of 5,376 patients agreed to participate in KORONA which is one of the largest nationwide RA cohorts in Korea. Patients were recruited between September 2009 and December 2011.

We defined the date of symptom onset as the date on which the patient first recognized joint pain or swelling, and the date of diagnosis as the date on which the patient was diagnosed with RA and received treatment based on common clinical practice by a physician. We defined early diagnosis as a lag time between symptom onset and an RA diagnosis of 12 months or shorter [4]. Patients who had a longer lag time were classified as the delayed diagnosis group. All patients provided informed consent under a protocol approved by the Institutional Review Board of each site.

\section{Functional disability and associated factors}

Functional disability was measured at enrollment with the Health Assessment Questionnaire-Disability Index (HAQ-DI) validated for use in Korean patients [14]. The range of HAQ-DI scores is between o and 3, where o represents no disability and 3 indicates complete disability. In our study, we defined no functional disability as HAQ-DI $=0$, since HAQ-DI in our data was strongly skewed to $O$ and clinically no disability was the goal of RA treatment (Fig. 1).

In order to find the factors associated with no functional disability (HAQ-DI = o), the demographic characteristics and clinical information were collected by detailed interviews and medical records, and disease activity was obtained by rheumatologists or well-trained health professionals at enrollment [15-17]. Since there were many patients with longer disease durations, to re-

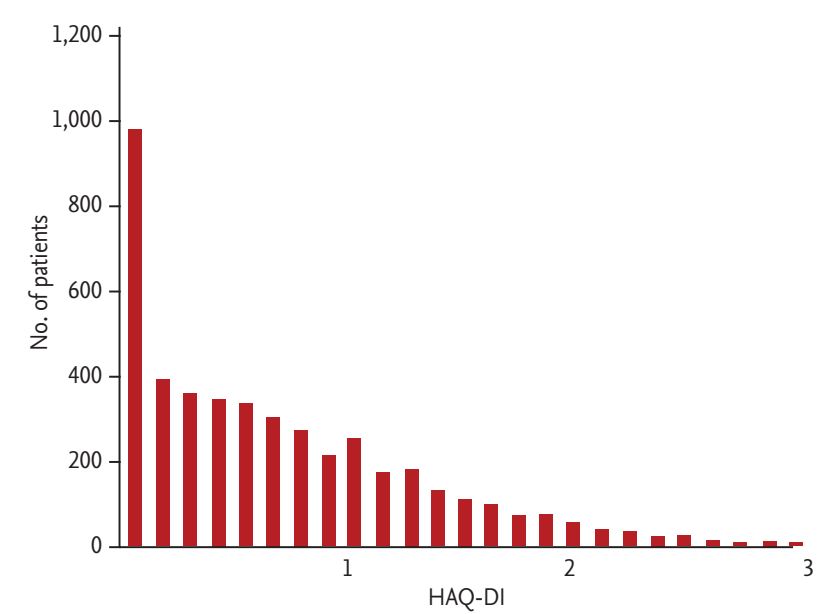

Figure 1. Distribution of Health Assessment QuestionnaireDisability Index (HAQ-DI) in this study. 
duce the recall bias about remote information including date of symptom onset and RA diagnosis, well-trained interviewer circumstantially asked patients about this information, accompanied by chart review in available patients.

Information on bone erosion at diagnosis was collected by rheumatologists or radiologists by determining whether bone erosion was present on hand and wrist radiographs using the radiographic image or description of bone erosion on medical chart of patients when they were diagnosed. Comorbidities were composed of prevalent diseases among RA patients including cardiovascular disease, hypertension, gastrointestinal disease, hepatitis, diabetes mellitus, thyroid disease, pulmonary disease, and malignancy.

\section{Statistical analysis}

In addition to demographic characteristics, clinical information, and disease activity, we compared the HAQDI score and the proportion of patients with no functional disability between early and delayed diagnosis groups, using the chi-square test and Student $t$ test. Multivariable logistic regression analysis was performed to identify the impact of early diagnosis on no functional disability, adjusting for variables such as age, sex, disease duration, education, absence of bone erosion, rheumatoid factor (RF) positivity, hemoglobin, disease activity score employing 28 joint-erythrocyte sedimentation rate (DAS28-ESR) and no comorbidity that were known to be associated with functional disability in previous studies [15-17] or significant in the crude analysis. The significance level was set at $p<0.05$.

Three separate logistic regression models were performed, according to disease duration, to analyze the impact of early diagnosis on functional disability of disease course. The disease durations tested, which defined as the duration between date of diagnosis and enrollment, were less than 5,5 to 10 , and $\geq 10$ years. For three separate logistic regression models, $p$ value adjusted for multiple comparisons $(p<0.0167$ corrected for three analysis) by the Bonferroni method was considered to be statistically significant. Statistical analyses were performed using SAS software version 9.2 (SAS, Cary, NC, USA).

\section{RESULTS}

\section{Demographic and clinical features}

A total of 2,597 patients (57.2\%) were included in the early diagnosis group and 1,943 patients (42.8\%) in the delayed diagnosis group. The demographic and clinical characteristics of the patients are listed in Table 1.

The mean delay before diagnosis was $3.5 \pm 3.7$ months in the early diagnosis group and $48.7 \pm 62.8$ months in the delayed diagnosis group. The early diagnosis group was younger $(52.8 \pm 12.1$ years vs. $54.5 \pm 12.2$ years, $p<0.01$ ) and its mean disease duration was longer than that of the delayed diagnosis group $(8.3 \pm 7.7$ years vs. $7.5 \pm 7.3$ years, $p$ $<0.01$ ). The early diagnosis group also had a higher educational level than the delayed diagnosis group $(59.5 \%$ vs. $53.8 \%$ with high school or higher, $p<0.01$ ).

In terms of functional disability, the average HAQ-DI score was higher in the delayed diagnosis group ( 0.64 \pm 0.63 vs. $0.70 \pm 0.66, p<0.01$ ), the mean difference between two groups was 0.06 , standard error 0.02 , and $95 \%$ confidence interval (CI) was 0.021 and 0.097 . The proportion of patients with no functional disability was higher in the early diagnosis group (22.9\% vs. $20.0 \%, p=$ 0.02 ), especially in those with disease duration less than 5 years (29.7\% vs. $23.8 \%, p<0.01$ ) (Fig. 2 ).

\section{Impact of early diagnosis on no functional disability}

Early diagnosis was associated with no functional disability (odds ratio [OR], 1.19; 95\% CI, 1.03 to 1.37) in crude analysis. In addition, several of the variables listed in Table 2 were also significantly associated with no functional disability.

After adjusting for variables that were significant in crude analysis, early diagnosis (OR, 1.19; 95\% CI, 1.01 to 1.40) remained associated with no functional disability. In addition, male gender (OR, 2.88; 95\% CI, 2.29 to 3.63), disease duration less than 5 years (OR, 1.42; 95\% CI, 1.16 to 1.75), higher level of education (OR, 1.70; $95 \% \mathrm{CI}, 1.39$ to 2.07), absence of bone erosion at diagnosis (OR, 1.42; 95\% CI, 1.20 to 1.68), RF positivity (OR, 1.49; 95\% CI, 1.13 to 1.98), and lack of comorbidity (OR, 1.30; 95\% CI, 1.09 to 1.54) were associated with no functional disability. The age (OR, 0.98; 95\% CI, 0.97 to 0.99 ) and DAS28-ESR (OR, $0.45 ; 95 \% \mathrm{CI}$, 0.42 to 0.49 ) were negatively associated with no functional disability. 
Table 1. Demographic and clinical characteristics of rheumatoid arthritis patients at enrollment

\begin{tabular}{|c|c|c|c|c|}
\hline Characteristic & Number & $\begin{array}{l}\text { Early diagnosis group } \\
(\mathrm{n}=2,597)\end{array}$ & $\begin{array}{l}\text { Delayed diagnosis group } \\
(\mathrm{n}=1,943)\end{array}$ & $p$ value \\
\hline Age, yr & 4,540 & $52.8 \pm 12.1$ & $54.5 \pm 12.2$ & $<0.01$ \\
\hline Male sex & 4,540 & $365(14.1)$ & $303(15.6)$ & 0.16 \\
\hline Disease duration, yr & 4,540 & & & 0.02 \\
\hline$<5$ & & $1,129(43 \cdot 5)$ & $923(47 \cdot 5)$ & \\
\hline $5 \leq$ and $<10$ & & $590(22.7)$ & $423(21.8)$ & \\
\hline$\geq 10$ & & $878(33.8)$ & $597(30.7)$ & \\
\hline Education & 4,520 & & & $<0.01$ \\
\hline Middle school or less & & $1,047(40.5)$ & $895(46.2)$ & \\
\hline High school or more & & $1,537(59 \cdot 5)$ & $1,041(53.8)$ & \\
\hline RF positivity & 4,540 & $2,357(90.8)$ & $1,743(89.7)$ & 0.26 \\
\hline Anti-CCP positivity & 3,522 & $1,726(84 \cdot 7)$ & $1,253(84.4)$ & 0.81 \\
\hline Hemoglobin, g/dL & 4,533 & $12.5 \pm 1.4$ & $12.5 \pm 1.4$ & 0.98 \\
\hline $\mathrm{ESR}, \mathrm{mm} / \mathrm{hr}$ & 4,540 & $29.1 \pm 23.8$ & $29.4 \pm 24.4$ & 0.68 \\
\hline $\mathrm{CRP}, \mathrm{mm} / \mathrm{dL}$ & 4,450 & $0.8 \pm 1.2$ & $0.8 \pm 1.3$ & 0.52 \\
\hline \multicolumn{5}{|c|}{ Extra-articular manifestations } \\
\hline Rheumatoid nodules & 4,539 & $99(3.8)$ & $74(3.8)$ & 1.00 \\
\hline Interstitial lung disease & 4,540 & $20(0.8)$ & $21(1.1)$ & 0.35 \\
\hline Bone erosion at diagnosis & 4,539 & $1,411(54 \cdot 3)$ & $1,090(56.2)$ & 0.21 \\
\hline Comorbidities positivity & 4,540 & $1,469(56.6)$ & $1,090(56.1)$ & 0.78 \\
\hline DAS28-ESR & 4,540 & $3.7 \pm 1.3$ & $3.7 \pm 1.4$ & 0.33 \\
\hline HAQ-DI score & 4,540 & $0.64 \pm 0.63$ & $0.70 \pm 0.66$ & $<0.01$ \\
\hline HAQ-DI = o & 4,540 & $594(22.9)$ & $388(20.0)$ & 0.02 \\
\hline Medications & 4,540 & & & \\
\hline MTX & & $2,157(83.1)$ & $1,620(83.4)$ & 0.81 \\
\hline Weekly dose, mg/wk & 3,769 & $12.6 \pm 2.9$ & $12.6 \pm 3.0$ & 0.99 \\
\hline Corticosteroids & & $1,947(75 \cdot 0)$ & $1,383(71.2)$ & $<0.01$ \\
\hline Daily dose, mg/day & 3,260 & $4.5 \pm 2.6$ & $4 \cdot 3 \pm 2.4$ & 0.12 \\
\hline Biologic agents & & $168(6.5)$ & $118(6.1)$ & 0.63 \\
\hline
\end{tabular}

Values are presented as mean \pm SD or number (\%).

RF, rheumatoid factor; CCP, cyclic citrullinated protein; ESR, erythrocyte sedimentation rate; CRP, C-reactive protein; DAS28-ESR, disease activity score employing 28 joint-erythrocyte sedimentation rate; HAQ-DI, Health Assessment Questionnaire-Disability Index; MTX, methotrexate.

\section{Impact of early diagnosis on no functional disability according to disease duration}

We also performed a subgroup analysis to analyze the influence of early diagnosis on no functional disability according to disease duration (Table 3). Early diagnosis was associated with no functional disability in patients with disease duration less than 5 years (OR, 1.37; 95\% CI, 1.09 to 1.72) but not in patients with longer disease dura- tion (for 5 to 10 years: OR, 1.07; 95\% CI, 0.75 to 1.52; for $\geq$ 10 years: OR, $0.92 ; 95 \% \mathrm{CI}, 0.65$ to 1.28 ).

In addition, no functional disability was affected by several variables along with the disease course. RF positivity (OR, 1.66; 95\% CI, 1.16 to 2.38) was associated with no functional disability only where disease duration was less than 5 years. On the other hands, absence of bone erosion at diagnosis (OR, 1.73; 95\% CI, 1.23 to 2.43 for $\geq 10$ 


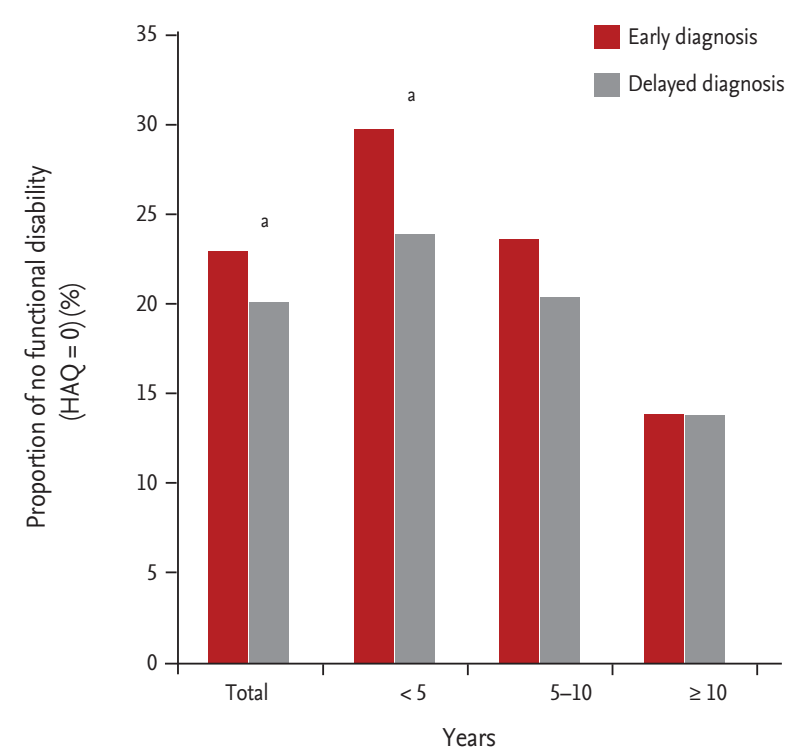

Figure 2. Proportion of patients with no functional disability (Health Assessment Questionnaire-Disability Index [HAQ-DI] score $=0$ ) in each group according to disease duration. ${ }^{a} p<0.05$, chi-square test. years) was associated with no functional disability where disease duration exceeded 10 years. Male gender (for $<5$ years: OR, 2.69; 95\% CI, 1.96 to 3.70; for 5 to 10 years: OR, 3.29; $95 \%$ CI, 2.03 to 5.32; for $\geq 10$ years: OR, 3.00; $95 \% \mathrm{CI}$, 1.86 to 4.85 ) and DAS28-ESR (for < 5 years: OR, $0.46 ; 95 \%$ CI, 0.41 to 0.51 ; for 5 to 10 years: OR, $0.41 ; 95 \% \mathrm{CI}, 0.34$ to 0.48; for $\geq 10$ years: OR, 0.47 ; $95 \% \mathrm{CI}$, 0.40 to 0.55 ) were consistently associated with no functional disability irrespective of disease duration.

\section{DISCUSSION}

In this study, the early diagnosis group showed lower HAQ-DI score, and a higher proportion of patients with no functional disability compared with delayed diagnosis group, though the differences were not large. We found that early diagnosis was independently associated with no functional disability after adjusting for various factors. In particular, early diagnosis was associated with no functional disability in patients with disease duration of less than 5 years, whereas no benefit of early diagno-

Table 2. Associated factors for no functional disability (HAQ-DI = 0 )

\begin{tabular}{|c|c|c|}
\hline Factor & Crude OR (95\% CI) & Multivariable OR (95\% CI) \\
\hline Age, yr & $0.97(0.96-0.97)^{\mathrm{a}}$ & $0.98(0.97-0.99)^{\mathrm{a}}$ \\
\hline Male sex & $3.42(2.87-4.07)^{\mathrm{a}}$ & $2.88(2.29-3.63)^{\mathrm{a}}$ \\
\hline Early diagnosis & $1.19(1.03-1.37)^{\mathrm{a}}$ & $1.19(1.01-1.40)^{\mathrm{a}}$ \\
\hline \multicolumn{3}{|l|}{ Disease duration, yr } \\
\hline$<5$ & $2.34(1.96-2.79)^{\mathrm{a}}$ & $1.42(1.16-1.75)^{\mathrm{a}}$ \\
\hline$\geq 5$ and $<10$ & $1.80(1.46-2.22)^{\mathrm{a}}$ & $1.25(0.99-1.59)$ \\
\hline$\geq 10$ & 1 & 1 \\
\hline \multicolumn{3}{|l|}{ Education } \\
\hline Middle school or less & 1 & 1 \\
\hline High school or more & $2.50(2.14-2.93)^{\mathrm{a}}$ & $1.70(1.39-2.07)^{\mathrm{a}}$ \\
\hline Absence of bone erosion at diagnosis & $1.94(1.68-2.24)^{\mathrm{a}}$ & $1.42(1.20-1.68)^{\mathrm{a}}$ \\
\hline RF positivity & $1.07(0.84-1.36)$ & $1.49(1.13-1.98)^{\mathrm{a}}$ \\
\hline Hemoglobin & $1.36(1.28-1.43)^{\mathrm{a}}$ & $0.95(0.89-1.02)$ \\
\hline DAS28-ESR & $0.43(0.40-0.46)^{\mathrm{a}}$ & $0.45(0.42-0.49)^{\mathrm{a}}$ \\
\hline No comorbidity & $1.62(1.41-1.87)^{\mathrm{a}}$ & $1.30(1.09-1.54)^{\mathrm{a}}$ \\
\hline
\end{tabular}

Multivariable OR was adjusted for age, sex, disease duration, education, absence of bone erosion, RF positivity, hemoglobin, DAS28-ESR, and no comorbidity.

HAQ-DI, Health Assessment Questionnaire-Disability Index; OR, odds ratio; CI, confidence interval; RF, rheumatoid factor; DAS28-ESR, disease activity score employing 28 joint-erythrocyte sedimentation rate.

a $p<0.05$. 
Table 3. Associated factors for no functional disability $(\mathrm{HAQ}-\mathrm{DI}=0$ ) according to disease duration

\begin{tabular}{lccc}
\hline \multirow{2}{*}{ Factor } & \multicolumn{3}{c}{ Disease duration, yr, OR $(95 \%$ CI $)$} \\
\cline { 2 - 4 } Age & $0.98(\mathrm{n}=2,052)$ & $5 \leq \mathrm{and}<10(\mathrm{n}=1,013)$ & $\geq 10(\mathrm{n}=1,475)$ \\
Male sex & $2.69(1.96-3.70)^{\mathrm{a}}$ & $0.98(0.97-1.00)$ & $0.98(0.96-0.99)^{\mathrm{a}}$ \\
Early diagnosis & $1.37(1.09-1.72)^{\mathrm{a}}$ & $3.29(2.03-5.32)^{\mathrm{a}}$ & $3.00(1.86-4.85)^{\mathrm{a}}$ \\
Education & & $1.07(0.75-1.52)$ & $0.92(0.65-1.28)$ \\
\multicolumn{1}{l}{ Middle school or less } & 1 & 1 & 1 \\
High school or more & $1.93(1.45-2.57)^{\mathrm{a}}$ & $1.44(0.94-2.21)$ & $1.50(1.02-2.21)$ \\
Absence of bone erosion at diagnosis & $1.25(0.99-1.58)$ & $1.50(1.06-2.11)$ & $1.73(1.23-2.43)^{\mathrm{a}}$ \\
RF positivity & $1.66(1.16-2.38)^{\mathrm{a}}$ & $1.68(0.84-3.39)$ & $0.97(0.54-1.74)$ \\
Hemoglobin & $0.94(0.85-1.03)$ & $0.97(0.85-1.11)$ & $0.99(0.86-1.13)$ \\
DAS28-ESR & $0.46(0.41-0.51)^{\mathrm{a}}$ & $0.41(0.34-0.48)^{\mathrm{a}}$ & $0.47(0.40-0.55)^{\mathrm{a}}$ \\
No comorbidity & $1.23(0.98-1.56)$ & $1.40(0.97-2.01)$ & $1.36(0.97-1.92)$ \\
\hline
\end{tabular}

Multivariable OR was adjusted for age, sex, education, absence of bone erosion, RF positivity, hemoglobin, DAS28-ESR, and no comorbidity.

HAQ-DI, Health Assessment Questionnaire-Disability Index; OR, odds ratio; CI, confidence interval; RF, rheumatoid factor; DAS28-ESR, disease activity score employing 28 joint-erythrocyte sedimentation rate.

a $p<0.0167$ (Bonferroni adjustment).

sis was observed in patients with disease duration more than 5 years. However, male gender and lower disease activity were consistently associated with no functional disability. In addition, absence of bone erosion at diagnosis was an independent factor for no functional disability where disease duration was longer than 10 years.

There have been several studies which assessed factors affecting functional outcome in RA patients [15-17]. They reported that several variables including age, gender, education, disease duration, disease activity, radiographic progression, and comorbidity were associated with functional disability; these results were comparable with our study. On the other hand, in our study, positive RF showed results opposite to those of previous study that reported positive RF was a predictor of worse functional disability [15]. Since positive RF is one of the criteria in RA classification [12,18], patients with positive RF tend to be diagnosed earlier and have greater possibility of receiving intensive treatment. Therefore, we assumed that positive RF could be associated with no functional disability when adjusting for variables including early diagnosis. However, further study for RF and functional status is needed to lead a conclusive result.

In our study, the mean difference of HAQ-DI score between early and delayed diagnosis group was o.o6; it is a relatively small difference, and does not exceed the minimally important difference (MID) for HAQDI from previous studies [19-23]. Considering that the mean HAQ-DI scores from previous studies were up to twice than ours, the MID of our study would be expected to be smaller than theirs. However, this difference of 0.06 might be clinically insignificant and thus additional study of MID in patients with similar baseline HAQDI is required.

Concerning the early diagnosis, a few previous studies have investigated the impact of early diagnosis on functional disability (Table 4) [8-11]. Two reported better outcomes for patients diagnosed early $[8,10]$, whereas others found similar outcomes between the early diagnosis and delayed diagnosis groups [9,11]. The patients examined in those studies were early RA patients who had never received DMARDs, and the numbers of patients investigated ranged from 40 to 608 . Two of these studies defined early diagnosis as a delay of less than 3 months from symptoms to diagnosis of RA [8,10]. The others classified patients into an early and a delayed treatment group according to the time between first visit and referral or referral and the start DMARDs [9,11]. The former pair of studies noted that HAQ scores improved more in the early diagnosis group and more patients in the early 


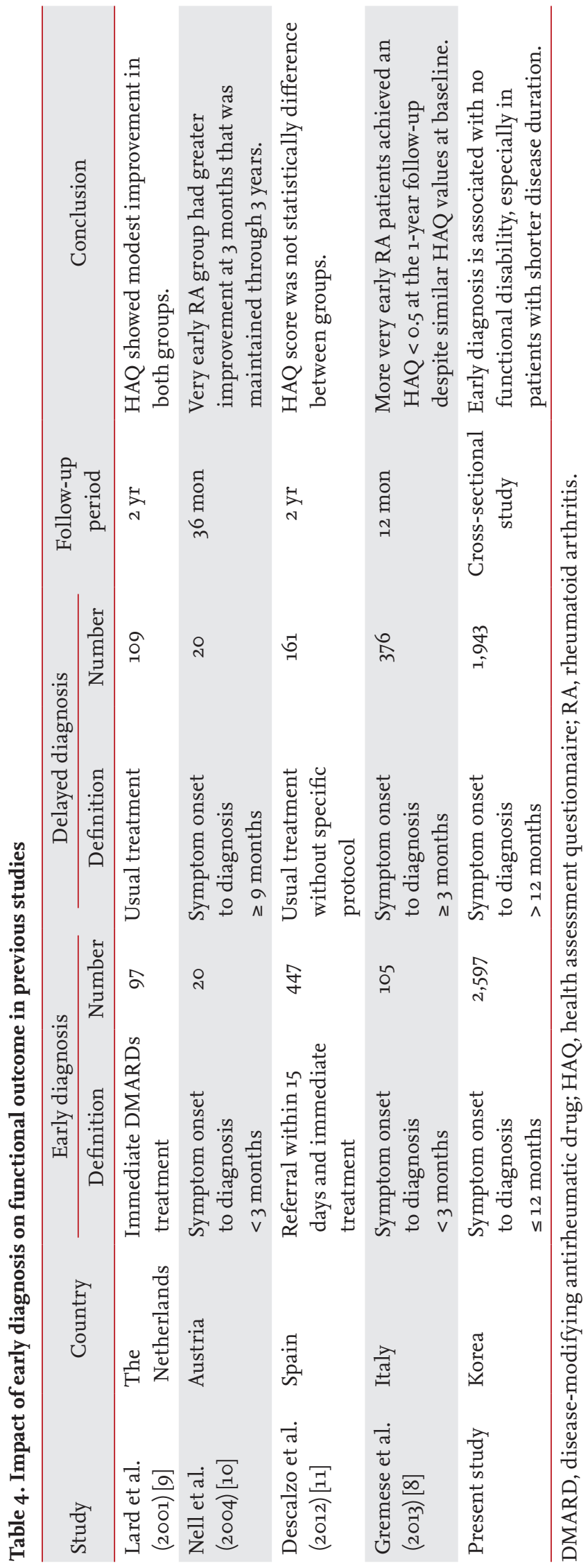

group achieved an HAQ score below 0.5 or 1.0 [8,10]. On the other hand, the latter pair of studies found only a modest improvement in HAQ scores, which, moreover, was similar in the two groups [9,11].

Our study differs in several respects from these previous studies. First, we used a large nationwide observational cohort that may better represent the actual clinical situation. Second, we included patients at all stages of the disease in order to reveal the effects of early diagnosis and treatment on functional disability with various disease durations. Third, the earlier studies examined the unadjusted effects on functional disability caused by early or delayed diagnosis; however, we adjusted for significant factors affecting HAQ-DI scores, namely age, gender, disease duration, level of education, initial joint damage, RF positivity, hemoglobin, disease activity, and comorbidity to identify the independent effect of early diagnosis on functional disability.

Interestingly, early diagnosis had an important influence on no functional disability over all disease durations, but in a subgroup analysis, the effect was only statistically significant for durations of less than 5 years. These results might be interpreted differently as a neglectable small effect of early diagnosis on functional disability, especially in disease duration longer than 5 years.

However, we can suggest three possible explanations for why the effect was restricted to patients with disease that had been present for less than 5 years. First, since age is a powerful determinant of functional disability [24], the impact of early diagnosis could be reduced in patients with longer disease duration because they tend to be older than those with shorter disease duration. Second, because we used a cross-sectional design, the common clinical practice of initial treatment was not identical according to the periods; the patients with long disease duration may have had less chance to receive aggressive treatments such as anti-tumor necrosis factor agents early in the course of their disease. Further study using detailed information about initial treatment could lead to more reliable results about early diagnosis and functional disability. Moreover, it was not possible to use time dependent variables in this analysis which can draw more reliable result about association of early diagnosis and functional disability along time, since this study was a cross-sectional study using enrollment 
data. We think we can draw a conclusive result when we analyze repetitive data using follow-up outcomes in future study using the prospective KORONA cohort. Third, since many of our patients had long disease durations, there may have been recall bias for symptom onset and date of diagnosis. The distinction between early and delayed diagnosis may therefore be more reliable in the case of patients with recent disease onset, but less reliable in the case of patients with disease of longer duration because of recall bias. This could have reduced the differences of functional disability between the early and delayed diagnosis groups among the patients with longer disease duration.

Despite the above limitations, our data show that early diagnosis is associated with no functional disability as well as other variables. A prospective cohort study of patients with relatively short disease durations of less than 5 years would allow the confirmation of the impact of early diagnosis on functional disability in the cases of longer disease durations.

In conclusion, early diagnosis was associated with no functional disability, especially in patients with shorter disease duration. On the other hand, absence of bone erosion at diagnosis was an independent factor for no functional disability where disease duration was longer than 10 years. Male gender and lower disease activity were consistently associated with no functional disability. Additional studies will be required to confirm these findings in the cases of longer disease durations. In addition, the difference of HAQ-DI score between early and delayed diagnosis group in this study was small; therefore, further study using patient population with various HAQ-DI distribution is needed to draw conclusive results.

\section{KEY MESSAGE}

1. Early diagnosis was associated with no functional disability, especially in patients with shorter disease duration.

2. Absence of bone erosion at diagnosis was an independent factor for no functional disability where disease duration was longer than 10 years.

3. Male gender and lower disease activity were consistently associated with no functional disability.

\section{Conflict of interest}

No potential conflict of interest relevant to this article was reported.

\section{Acknowledgments}

This study was supported by the Korea Healthcare Technology R\&D Project, Ministry of Health and Welfare, Republic of Korea (HI10C2O2O). The authors wish to acknowledge the assistance of the following investigators who enrolled patients in KORONA: Joong Kyoung Ahn (Samsung Medical Center, Sungkyunkwan University School of Medicine, Seoul), Sung Jae Choi (Korea University Ansan Hospital, Ansan), Minyoung Her (Inje University Busan Paik Hospital, Busan), Chung-Il Joung (Konyang University Hospital, Daejeon), Young Mo Kang (Kyungpook National University Hospital, Daegu), Dong-Yook Kim (Inje University Busan Paik Hospital, Busan), Hae-Rim Kim (Konkuk University Medical Center, Seoul), Hyoun Ah Kim (Ajou University Hospital, Suwon), Choong Ki Lee (Yeungnam University Medical Center, Daegu), Sang-Hoon Lee (Kyung Hee University Hospital at Gangdong, Seoul), Seong-Su Nah (Soon Chun Hyang University Cheonan Hospital, Cheonan), Sung-Hoon Park (Catholic University of Daegu School of Medicine, Daegu), Dong Hyuk Sheen (Eulji University Hospital, Daejeon), Gwan Gyu Song (Korea University Guro Hospital, Seoul), Chang-Hee Suh (Ajou University Hospital, Suwon), and Yoo Wan-Hee (Chonbuk National University Hospital, Jeonju).

\section{REFERENCES}

1. Breedveld F. The value of early intervention in RA: a window of opportunity. Clin Rheumatol 2011;30 Suppl 1:S33-S39.

2. Cush JJ. Early rheumatoid arthritis: is there a window of opportunity? J Rheumatol Suppl 2007;80:1-7.

3. Bosello S, Fedele AL, Peluso G, Gremese E, Tolusso B, Ferraccioli G. Very early rheumatoid arthritis is the major predictor of major outcomes: clinical ACR remission and radiographic non-progression. Ann Rheum Dis 2011;70:1292-1295.

4. Kyburz D, Gabay C, Michel BA, Finckh A; physicians of SCQM-RA. The long-term impact of early treatment of rheumatoid arthritis on radiographic progression: a 
population-based cohort study. Rheumatology (Oxford) 2011;50:1106-1110.

5. Lukas C, Combe B, Ravaud P, Sibilia J, Landew R, van der Heijde D. Favorable effect of very early disease-modifying antirheumatic drug treatment on radiographic progression in early inflammatory arthritis: data from the Étude et Suivi des polyarthrites indifférenciées récentes (study and followup of early undifferentiated polyarthritis). Arthritis Rheum 2011;63:1804-1811.

6. Finckh A, Liang MH, van Herckenrode CM, de Pablo P. Long-term impact of early treatment on radiographic progression in rheumatoid arthritis: a meta-analysis. Arthritis Rheum 2006;55:864-872.

7. Mottonen T, Hannonen P, Korpela M, et al. Delay to institution of therapy and induction of remission using single-drug or combination-disease-modifying antirheumatic drug therapy in early rheumatoid arthritis. Arthritis Rheum 2002;46:894-898.

8. Gremese E, Salaffi F, Bosello SL, et al. Very early rheumatoid arthritis as a predictor of remission: a multicentre real life prospective study. Ann Rheum Dis 2013;72:858862.

9. Lard LR, Visser H, Speyer I, et al. Early versus delayed treatment in patients with recent-onset rheumatoid arthritis: comparison of two cohorts who received different treatment strategies. Am J Med 2001;111:446-451.

10. Nell VP, Machold KP, Eberl G, Stamm TA, Uffmann M, Smolen JS. Benefit of very early referral and very early therapy with disease-modifying anti-rheumatic drugs in patients with early rheumatoid arthritis. Rheumatology (Oxford) 2004;43:906-914.

11. Descalzo MA, Carbonell J, Gonzalez-Alvaro I, et al. Effectiveness of a clinical practice intervention in early rheumatoid arthritis. Arthritis Care Res (Hoboken) 2012;64:321330.

12. Arnett FC, Edworthy SM, Bloch DA, et al. The American Rheumatism Association 1987 revised criteria for the classification of rheumatoid arthritis. Arthritis Rheum 1988;31:315-324.

13. Sung YK, Cho SK, Choi CB, et al. Korean Observational Study Network for Arthritis (KORONA): establishment of a prospective multicenter cohort for rheumatoid arthritis in South Korea. Semin Arthritis Rheum 2012;41:745-751.

14. Bae SC, Cook EF, Kim SY. Psychometric evaluation of a
Korean Health Assessment Questionnaire for clinical research. J Rheumatol 1998;25:1975-1979.

15. Welsing PM, van Gestel AM, Swinkels HL, Kiemeney LA, van Riel PL. The relationship between disease activity, joint destruction, and functional capacity over the course of rheumatoid arthritis. Arthritis Rheum 2001;44:20092017.

16. Escalante A, del Rincon I. How much disability in rheumatoid arthritis is explained by rheumatoid arthritis? Arthritis Rheum 1999;42:1712-1721.

17. Cho SK, Sung YK, Choi CB, et al. Do patients with elderly-onset rheumatoid arthritis have severe functional disability? Semin Arthritis Rheum 2012;42:23-31.

18. Aletaha D, Neogi T, Silman AJ, et al. 2010 Rheumatoid arthritis classification criteria: an American College of Rheumatology/European League Against Rheumatism collaborative initiative. Arthritis Rheum 2010;62:25692581.

19. Wells GA, Tugwell P, Kraag GR, Baker PR, Groh J, Redelmeier DA. Minimum important difference between patients with rheumatoid arthritis: the patient's perspective. J Rheumatol 1993;20:557-560.

20. Redelmeier DA, Lorig K. Assessing the clinical importance of symptomatic improvements: an illustration in rheumatology. Arch Intern Med 1993;153:1337-1342.

21. kosinski M, Zhao SZ, Dedhiya S, Osterhaus JT, Ware JE Jr. Determining minimally important changes in generic and disease-specific health-related quality of life questionnaires in clinical trials of rheumatoid arthritis. Arthritis Rheum 2000;43:1478-1487.

22. Greenwood MC, Doyle DV, Ensor M. Does the Stanford Health Assessment Questionnaire have potential as a monitoring tool for subjects with rheumatoid arthritis? Ann Rheum Dis 2001;60:344-348.

23. Pope JE, Khanna D, Norrie D, Ouimet JM. The minimally important difference for the health assessment questionnaire in rheumatoid arthritis clinical practice is smaller than in randomized controlled trials. J Rheumatol 2009;36:254-259.

24. Sokka T, Krishnan E, Hakkinen A, Hannonen P. Functional disability in rheumatoid arthritis patients compared with a community population in Finland. Arthritis Rheum 2003;48:59-63. 